\title{
Pattern of brain injury and depressed heart rate variability in newborns with hypoxic ischemic encephalopathy
}

\author{
Marina Metzler ${ }^{1}$, Rathinaswamy Govindan ${ }^{1}$, Tareq Al-Shargabi ${ }^{1}$, Gilbert Vezina ${ }^{2}$, Nickie Andescavage ${ }^{3}$, Yunfei Wang ${ }^{4}$,
} Adre du Plessis ${ }^{1}$ and An N. Massaro ${ }^{3}$

BACKGROUND: Decreased heart rate variability (HRV) is a measure of autonomic dysfunction and brain injury in newborns with hypoxic ischemic encephalopathy (HIE). This study aimed to characterize the relationship between HRV and brain injury pattern using magnetic resonance imaging (MRI) in newborns with HIE undergoing therapeutic hypothermia.

METHODS: HRV metrics were quantified in the time domain $\left(a_{S}, a_{L}\right.$, and root mean square at short $\left(R M S_{S}\right)$ and long $\left(R M S_{L}\right)$ timescales) and frequency domain (relative low-(LF) and highfrequency (HF) power) over 24-27 h of life. The brain injury pattern shown by MRI was classified as no injury, pure cortical/ white matter injury, mixed watershed/mild basal ganglia injury, predominant basal ganglia or global injury, and death. HRV metrics were compared across brain injury pattern groups using a random-effects mixed model.

RESULTS: Data from 74 infants were analyzed. Brain injury pattern was significantly associated with the degree of HRV suppression. Specifically, negative associations were observed between the pattern of brain injury and RMS (estimate -0.224 , SE 0.082, $P=0.006$ ), RMS (estimate -0.189 , SE 0.082, $P=0.021$ ), and LF power (estimate -0.044 , SE 0.016, $P=0.006$ ). CONCLUSION: Degree of HRV depression is related to the pattern of brain injury. HRV monitoring may provide insights into the pattern of brain injury at the bedside.

H ypoxic ischemic encephalopathy (HIE) is a major cause of death and disability in newborns. Although therapeutic hypothermia has improved outcome in infants with moderate and severe encephalopathy, nearly half of these infants continue to have adverse outcomes (1). Biomarkers that can provide early insights to help specify the type and pattern of brain injury can provide opportunities for targeted therapies and aid in prognostication.

Currently, magnetic resonance imaging (MRI) is the standard for the assessment of brain injury in newborns with HIE $(2,3)$. It has been well established that the pattern of injury shown by MRI relates to later developmental outcomes
(4-8). Specifically, injury to the basal ganglia and thalamic brain regions has been associated with poor neuromotor outcomes $(4,5)$, whereas cortical watershed (WS)-type injuries relate to school-aged cognitive deficits, particularly lower language-related abilities (6). Although MRI provides detailed information on the location of injury, its sensitivity for reflecting the extent of brain injury in the first $24 \mathrm{~h}$ of life has been questioned (4). Therefore, its use is generally restricted to the subacute period after completion of therapeutic hypothermia when there are fewer factors limiting transport of the critically ill newborn to the scanner. Thus, biomarkers that reflect the pattern of brain injury after hypoxia-ischemia that can be measured during hypothermia treatment can provide key information to the bedside clinician that is currently lacking.

Heart rate variability (HRV) offers a continuous and quantitative method to assess the autonomic nervous system (ANS) non-invasively and has been proposed as a bedside biomarker in newborns with HIE (9-12). Prior studies have shown that reduced HRV is associated with severity of brain injury as shown by MRI (9), abnormal electroencephalogram findings $(9,10)$, and poor long-term neurodevelopmental outcome (10-12) in infants with moderate to severe encephalopathy. These studies have related HRV metrics to the presence and severity of brain injury, but not to the topography of injury. Recently, we demonstrated that injury at both the cerebral cortical and brain stem levels was associated with ANS dysregulation in newborns with various types of brain injury (13). These findings suggest that autonomic regulation can be mediated by both direct injury to the deep-brain structures including the brainstem as well as to higher-order cortical centers. The aim of this study was to investigate whether HRV is related to the topography of brain injury shown by MRI in newborns with HIE. We hypothesized that HRV depression would be observed in infants with WS-predominant brain injury compared to infants with normal MRI and that the greatest HRV depression would be observed in infants with a basal ganglia/thalamus (BG)predominant pattern of injury.

\footnotetext{
${ }^{1}$ Division of Fetal and Transitional Medicine, Children's National Health System, Washington, DC; ${ }^{2}$ Division of Diagnostic Imaging and Radiology, Children's National Health System, Washington, DC; ${ }^{3}$ Divsion of Neonatology, Children's National Health System, Washington, DC; ${ }^{4}$ Division of Biostatistics and Study Methodology, Children's National Health System, Washington, DC. Correspondence: An N. Massaro (anguyenm@childrensnational.org) 


\section{RESULTS}

Of the 80 infants enrolled, 74 had electrocardiogram (EKG) data available during the study period. The remaining infants were excluded because of unavailability of data $(n=4)$ and death before $24 \mathrm{~h}$ of life $(n=2)$. MRI used for classification was performed at a median age of 10 days (range 3-18). Early MRI was used for classification in 14 infants who did not undergo late MRI because of death or discharge prior to the target window. The distribution of brain injury pattern in order of increasing severity was as follows: 34 infants had no injury (46\%); 10 had a pure cortical/white matter injury (WMI) pattern (14\%); 6 had a mixed WS/mild BG pattern (8\%); 15 had a predominant BG or global injury pattern (20\%); and 9 died (12\%). Clinical characteristics of the study population are presented in Table 1.

\section{Detrended Fluctuation Analysis}

In bivariate analyses, there were significant associations $(P<0.001)$ between brain injury pattern and all detrended fluctuation analysis metrics (Figure 1). Reduced HRV in the injured groups was shown by a decrease in the value of $\alpha_{S}$, $\mathrm{RMS}_{\mathrm{S}}$, and $\mathrm{RMS}_{\mathrm{L}}$ and an increase in the value of $\alpha_{\mathrm{L}}$ across the increasing injury severity groups. In the model adjusted for covariates, the brain injury pattern remained significantly associated with $\mathrm{RMS}_{\mathrm{S}}$ (estimate -0.224 , SE $0.082, P=0.006$ ) and $\mathrm{RMS}_{\mathrm{L}}$ (estimate -0.189 , SE $0.082, P=0.021$ ). Encephalopathy grade was also significantly associated with HRV metrics across models $(P<0.05)$.

\section{Spectral Analysis}

A decrease in relative low-frequency (LF) power and an increase in relative high-frequency (HF) power was observed across brain injury pattern groups (Figure $2 \mathbf{a}, \mathbf{b}, P<0.001$ ). Bivariate associations were similarly significant when considering absolute LF and HF power, although absolute HF power decreased across brain injury pattern groups (Figure $2 \mathrm{c}, \mathbf{d}, P<0.001)$. After adjusting for covariates, there was a significant negative association between brain injury pattern and relative LF power (estimate -0.044 , SE 0.016, $P=0.006$ ). Encephalopathy grade was also significantly inversely associated with relative LF power and directly associated with relative HF power $(P<0.05)$.

Impact of Seizures on Brain Injury Pattern and HRV Association Nine infants were subsequently excluded for electrographic seizures during the study period: two with pure cortical/WMI, four with mixed WS/mild BG, one with predominant BG or global injury pattern, and two who died. The associations between brain injury pattern and HRV metrics $\mathrm{RMS}_{\mathrm{S}}$ (estimate $-0.225, \mathrm{SE} 0.088, P=0.011$ ), $\mathrm{RMS}_{\mathrm{L}}$ (estimate -0.198 , SE 0.086, $P=0.022$ ), and LF power (estimate -0.050 , SE $0.017, P=0.004)$ were similar across these reduced models.
Table 1. Clinical characteristics

\begin{tabular}{|c|c|}
\hline $\begin{array}{l}\text { Gestational age, weeks (mean } \pm \text { SD) } \\
\text { Birth weight, kg (mean } \pm S D)\end{array}$ & $\begin{array}{c}38.7 \pm 1.5 \\
3.3 \pm 0.7\end{array}$ \\
\hline Male gender $(n, \%)$ & $37(50)$ \\
\hline \multicolumn{2}{|l|}{ Apgar score } \\
\hline 1 min (median, range) & $1(0-9)$ \\
\hline $5 \min ^{\mathrm{a}}$ (median, range) & $3(0-9)$ \\
\hline $10 \min ^{\mathrm{b}}$ (median, range) & $5(0-10)$ \\
\hline Initial pH (median, range) & $6.95(6.50-7.42)$ \\
\hline \multicolumn{2}{|c|}{ Encephalopathy grade at presentation (modified Sarnat) } \\
\hline Moderate $(n, \%)$ & $55(74)$ \\
\hline Severe $(n, \%)$ & $19(26)$ \\
\hline EEG seizures $(n, \%)$ & $9(12)$ \\
\hline Received phenobarbital $(n, \%)$ & $19(26)$ \\
\hline Received vasoactives ( $\mathrm{n}, \%)$ & $33(45)$ \\
\hline Dopamine $(n, \%)$ & $33(45)$ \\
\hline Epinephrine $(n, \%)$ & $4(5)$ \\
\hline Age at MRI (median, range) ${ }^{c}$ & $10(3-18)$ \\
\hline Respiratory support (n, \%) & $66(89)$ \\
\hline High-frequency ventilator $(n, \%)$ & $7(9)$ \\
\hline Conventional ventilator $(n, \%)$ & $39(53)$ \\
\hline Other $(n, \%)$ & $20(27)$ \\
\hline None $(n, \%)$ & $8(11)$ \\
\hline \multicolumn{2}{|l|}{ Respiratory rate } \\
\hline $\begin{array}{l}\text { Conventional ventilator (median, interquartile } \\
\text { range) }\end{array}$ & $22(16-28)$ \\
\hline $\begin{array}{l}\text { Spontaneous breathing (median, interquartile } \\
\text { range) }\end{array}$ & $31(25-36)$ \\
\hline High Frequency Ventilator & $\mathrm{n} / \mathrm{a}$ \\
\hline Heart rate overall (median, interquartile range) & $110(99-128)$ \\
\hline Group 0 & $103(96-124)$ \\
\hline Group 1 & $109(96-120)$ \\
\hline Group 2 & $106(100-135)$ \\
\hline Group 3 & $116(107-131)$ \\
\hline Group 4 & $127(114-145)$ \\
\hline \multicolumn{2}{|c|}{ 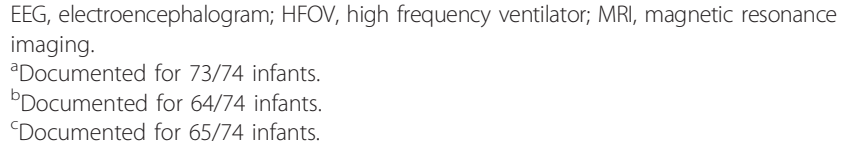 } \\
\hline
\end{tabular}

\section{HRV Prediction of BG Injury}

Receiver operating curve analyses were performed to determine optimal cutoff points of $\mathrm{RMS}_{\mathrm{S}}, \mathrm{RMS}_{\mathrm{L}}$, and relative LF power for distinguishing infants who died or had significant BG injury (Groups 3 and 4) from those with no BG injury (Groups 0 and 1). Area under the receiver operating curve and predictive abilities are summarized in Table 2. 


\section{Articles | Metzler et al.}

\section{DISCUSSION}

Brain injury pattern is associated with reduced $\mathrm{HRV}$ at $24 \mathrm{~h}$ of life in newborns with HIE who are undergoing therapeutic hypothermia. As expected, presence of global or BG injury was associated with significant HRV depression compared with absence of brain injury. Reduced HRV was also observed with cortical/WMI alone, although to a lesser degree than that a
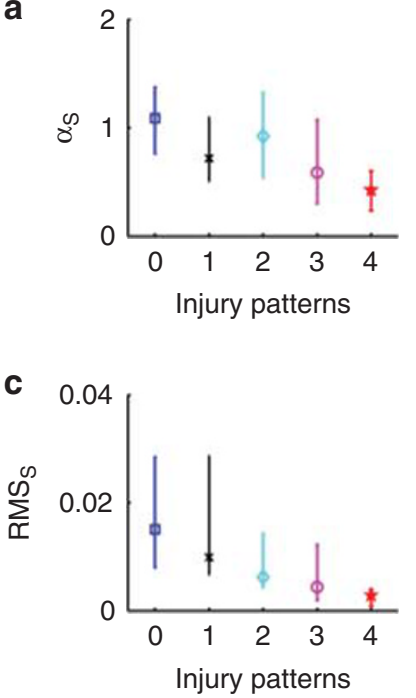

b

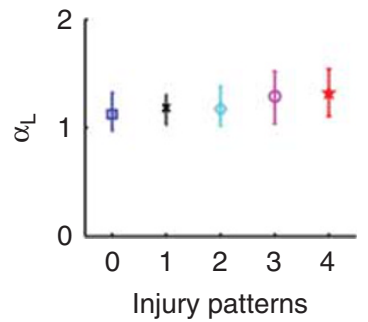

d

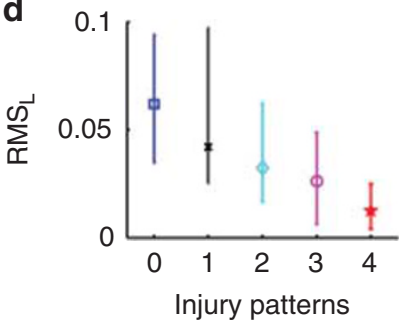

Figure 1. DFA metrics. DFA metrics are as follows: (a) $a_{S,}$ (b) $a_{L}$, (c) $\mathrm{RMS}_{\mathrm{S}}$, and (d) $\mathrm{RMS}_{\mathrm{L}}$ presented as the median (interquartile range). Injury pattern groups are defined as follows: $0=$ no injury; $1=$ pure cortical/ WMI; 2 =mixed WS/mild BG; $3=$ predominant BG or global injury; and 4 = died. BG, basal ganglia/thalamus; DFA, detrended fluctuation analysis; $\mathrm{RMS}_{\mathrm{L}}$, root mean square at long timescales; $\mathrm{RMS}_{\mathrm{S}}$, root mean square at short timescales; WMI, white matter injury; WS, watershed. seen with global or BG injury. Brain injury pattern was most associated with HRV metrics reflecting the sympathetic component of the ANS $\left(\mathrm{RMS}_{S}, \mathrm{RMS}_{\mathrm{L}}\right.$, and $\left.\mathrm{LF}\right)$. These relationships remained significant after adjusting for encephalopathy grade at presentation and after excluding patients with electrographic seizures. These results suggest that monitoring HRV during therapeutic hypothermia may provide bedside insights into the topography of brain injury in newborns with HIE.

Our findings of reduced HRV in infants with brain injury are consistent with other studies of HRV in infants with HIE (9-12). In particular, LF power has been associated with adverse neuroimaging and functional outcomes (10-12). However, our study is the first to evaluate the association between HRV metrics and pattern of injury, not just presence of injury. This functionality may have important clinical implications, as it has been suggested that pattern of injury shown by MRI is more predictive of outcome than assessment of severity of injury to a particular region alone (5). It is recognized, however, that our findings may reflect the relationship between HRV and gradations of overall brain injury severity, as patterns of injury may represent a

Table 2. Heart rate variability prediction of basal nucleus injury

\begin{tabular}{lccccc}
\hline HRV metric & Sensitivity & Specificity & PPV & NPV & AUC \\
\hline RMS $_{S}$ & 0.609 & 0.865 & 0.723 & 0.792 & 0.788 \\
RMS $_{L}$ & 0.678 & 0.750 & 0.734 & 0.696 & 0.771 \\
rLF & 0.717 & 0.680 & 0.740 & 0.655 & 0.776 \\
\hline
\end{tabular}

AUC, area under the receiver operating curve; HRV, heart rate variability; NPV, negative predictive value; PPV, positive predictive value; $r L F$, relative low-frequency power; RMS , root mean square at long timescales; $\mathrm{RMS}_{S}$, root mean square at short timescales.
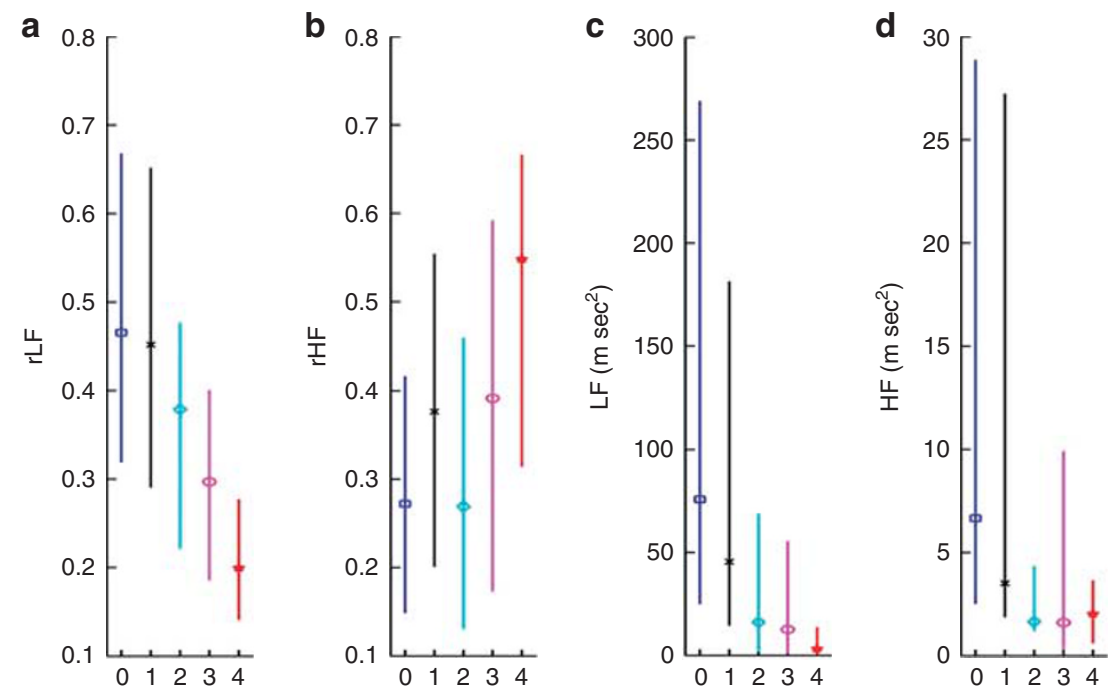

Figure 2. SA metrics. SA metrics are as follows: (a) rLF and (b) rHF power presented as median (interquartile range). Absolute (c) LF and (d) HF powers are also shown for comparison. Injury pattern groups are defined as follows: $0=$ no injury; $1=$ pure cortical $/ \mathrm{WMl} ; 2=\mathrm{mixed} \mathrm{WS} / \mathrm{mild} \mathrm{BN}$; $3=$ predominant $\mathrm{BG}$ or global injury; and 4 = died. BG, basal ganglia/thalamus; rHF, relative high frequency; rLF, relative low frequency; SA, spectral analysis; WMI, white matter injury; WS, watershed. 
continuum of injury severity. Similarly, the timing of our assessment of cortical injury shown by MRI may limit our ability to make firm conclusions about cortical injury in vivo and its relation to HRV in real time. However, that these findings could reflect a mechanistic link between regional injury and cerebral inputs regulating autonomic control of heart rate is consistent with animal and human studies that have described the role of the cortical autonomic network in the regulation of HRV $(14,15)$. We speculate that the depression of HRV in infants with isolated cortical injury supports the notion that cerebral cortical inputs are involved in the regulation of the ANS by term gestation (13). Although our findings cannot confirm this mechanistic link, these data can provide ranges for HRV metrics that can reflect the risk for basal ganglia/thalamic injury (BG) vs. WS involvement for the bedside clinician.

The relationship between HRV and topography of brain injury further strengthens its utility as a bedside monitoring tool. Although MRI provides high anatomical resolution, its limited accessibility during the first $24 \mathrm{~h}$ of critical illness limits its temporal resolution (4). Despite improved access in neonatal intensive care units experienced with transport to the MRI suite, its use remains impracticable in the most critically ill patients. HRV analysis, alternatively, allows for real-time assessment of injury severity, and possibly topography of injury, at the bedside. These insights could be helpful to direct therapies for individual patients during the acute and subacute periods, optimizing opportunities for intervention within the therapeutic window.

It has been suggested that topography of injury may indicate the etiology of particular insults. For example, whereas a global or BG-predominant pattern has been associated with acute profound asphyxia $(5,16,17)$, cortical and focal/multifocal WMI has been more commonly described in patients with accompanying chorioamnionitis or placental insufficiency (18). As these variable insults may be amenable to alternative neuroprotective strategies, early stratification of patients by brain injury pattern can have therapeutic implications. Brain injury pattern may in itself indicate responsiveness to cooling, as it has been suggested that alternative cooling regimens may be selectively neuroprotective for cortical vs. deep brain structures (19-21). Pattern of injury is also important for prognostication. Basal ganglia and/or thalamic injury have been associated with adverse motor outcomes and cerebral palsy $(5,17)$. Conversely, injury to the WS regions has been associated with cognitive deficits at 30 months of age (5) as well as at 4 (ref. 6) and 11 years of age (22). Thus, early insights into the pattern of injury may guide expectations for developmental phenotypes (5). We have not yet correlated our brain injury patterns with neurodevelopmental outcome. This correlation is needed and assessment is ongoing. Future analyses will need to assess the additive value of HRV monitoring over standard bedside assessments including clinical examination and electroencephalogram.
This study has several limitations. As the exact mechanisms mediating HRV after a hypoxic ischemic insult are complex, the covariates we chose are not all encompassing. For example, intrinsic myocardial dysfunction may be an important factor in HRV depression in babies with HIE. Although we adjusted for vasopressor use, we did not account for the dose or cumulative amount of vasopressors required, nor for other indices of cardiovascular instability. Similarly, it is recognized that the respiratory oscillations can have an impact on heart rate and HRV (23). Given the neonatal respiratory rate falls within the HF band interrogated, the relationship between HF power and brain injury observed in our unadjusted analyses may reflect increasing ventilator dependence in the infants with more severe brain injury. We are unable to remove ventilator-related oscillations from the heart rate. Whereas previous studies have used the spectral power around the breathing frequency as the representation of HF power $(23,24)$, this approach focuses on quantifying ventilator-related oscillations rather than all the components of parasympathetic tone that are believed to reside within the more conventionally defined HF band used in this work. While we are developing methods to isolate respiratory oscillations to remove these effects from quantification of HRV in the HF band, we have to consider the effect of the ventilator oscillations on heart rate as a limitation of this study. As mentioned, our study is unable to differentiate association and causation. Whether regional injury differentially influences autonomic function is unclear; however, these data do provide estimates of HRV metrics that correlate with different patterns of brain injury. Although we entered into statistical analyses with a large number of observations given the repeated measures over time in subjects, we acknowledge that the sample size was limited, particularly in the mixed cortical/mild BN group. Larger studies will need to confirm these findings in order to provide practical benchmark ranges that can signify brain injury categories with reliability. Generalizability of these analyses may be affected by the distribution of injury pattern; however, the frequencies of different injury patterns were comparable to other studies in newborns with HIE treated with hypothermia $(8,25)$. Finally, it should be recognized that MRI was the tool used for assessment of brain injury in this study and that these findings may differ when considering long-term developmental outcome phenotypes.

\section{CONCLUSIONS}

Pattern of brain injury relates to the degree of HRV depression in newborns with HIE. Evaluation of the degree and pattern of brain injury may be aided by real-time HRV monitoring in HIE infants undergoing therapeutic hypothermia.

\section{METHODS}

\section{Study Population}

Infants with HIE undergoing therapeutic hypothermia were included from a prospective observational study evaluating biomarkers of brain injury. Inclusion criteria were based on the modified National Institute of Child Health and Human Development criteria: gestational age at birth $\geq 35$ weeks, birth weight $\geq 1,800$ g, metabolic 


\section{Articles $\mid$ Metzler et al.}

acidosis and/or low Apgar score in the setting of a perinatal sentinel event, and moderate to severe encephalopathy based on modified Sarnat criteria (1). Infants were systemically cooled to $33.5^{\circ} \mathrm{C}$ for $72 \mathrm{~h}$ and rewarmed to $0.5^{\circ} \mathrm{C} / \mathrm{h}$ over $6 \mathrm{~h}$ (Blanketrol II, Cincinnati Sub-Zero, Cincinnati, OH). The Children's National Health System Institutional Review Board approved the study, and informed consent was obtained from the parent of each participant.

\section{Data Collection}

Clinical and demographic data were collected from the birth hospital and study-site medical records. Continuous EKG recordings were obtained from the bedside cardiorespiratory monitor (Philips IntelliVue MP70, Andover, MA). For infants enrolled before $24 \mathrm{~h}$ of life, EKG recordings were prospectively collected at a sample rate of $1,000 \mathrm{~Hz}$ using a custom software developed in LabView (National Instruments, Austin, TX). Otherwise, EKG data were collected if available from an institutional Research Data Export archive (IntelliVue Information Center, Philips Healthcare, Andover, MA) at a sample rate of $125 \mathrm{~Hz}$. We focused on EKG data from 24 to $27 \mathrm{~h}$ of life based on our prior work, demonstrating this as a key time period during which HRV best discriminates between outcome groups (11).

\section{EKG Processing}

To attenuate the noise and to correct for the baseline shifts, the EKG was bandpass-filtered between 0.5 and $60 \mathrm{~Hz}$ using a Butterworth filter with zero-phase distortion. The R-wave (a wave with maximum amplitude of the cardiac cycle) was identified using a combination of Hilbert transform and the adaptive threshold detection approach (26), and beat-to-beat interval (RRi) was calculated. The artifacts in the RRi were cleaned using a recently proposed data-driven approach (27). The artifact-free RRi's were partitioned into 10-min epochs. All analyses were performed offline using MATLAB (MathWorks, Natick, MA).

\section{Detrended Fluctuation Analysis}

Detrended fluctuation analysis involves the following four steps: (i) removing the average value of the data and calculating the profile function, which is a cumulative sum of the data; (ii) dividing the profile function into time windows, each containing $s$ - number of beats; (iii) fitting a polynomial function to the profile inside each window and calculating the local fluctuation as the root mean square deviation of the profile from the best fit; (iv) averaging the local fluctuation function over all windows to get an overall fluctuation function. For power-law-correlated signals such as RRi, the fluctuation function varies with the window size $s$. The fluctuation exponent is calculated as the slope of the fluctuation function. In this work, we used a fourth-order polynomial to detrend the RRi and calculated the following metrics: $\alpha_{S}$ (15-50 beats), $\alpha_{L}(100-150$ beats), $\mathrm{RMS}_{\mathrm{S}}$ (15-50 beats), and $\mathrm{RMS}_{\mathrm{L}}$ (100-150 beats); (ref. 28$)$. The $\alpha$ metrics characterize the autocorrelations in the data, whereas the RMS metrics characterize the variability in the data. For less variable data, RMS and $\alpha_{S}$ values will be low, and for more redundant data the $\alpha_{\mathrm{L}}$ metric value will be high.

\section{Spectral Analysis}

For spectral analysis, the RRis in each 10-min epoch were interpolated using cubic spline with a sample rate of $4 \mathrm{~Hz}$ and converted into evenly sampled data. To estimate the power spectrum of RRi in a 10-min window, we used a Welch periodogram approach with a frequency resolution of $0.016 \mathrm{~Hz}$. In this approach, we partitioned the RRi into 1-min nonoverlapping epochs. For the RRi in each epoch, we subtracted the mean and normalized it by the SD to mitigate the effects of non-stationarity as described previously (29). In each window, we calculated the periodogram as the square of the magnitude of the Fourier transform of the RRi. To obtain an estimate of the power spectrum, we averaged the periodograms over all 1-min epochs. The sum of powers in LF $(0.05-0.25 \mathrm{~Hz})$ and HF $(0.3-1 \mathrm{~Hz})$ bands was calculated. The normalized or relative powers were calculated by dividing the spectral powers by the total power in order to minimize the effect of changes in total power on the estimations of LF and HF components (30). The total power was defined as the sum of powers in the frequency band of $0.05-2 \mathrm{~Hz}$ $(11,28,29)$.

In summary, reduced HRV is associated with lower values for the HRV metrics $\alpha_{S}, \mathrm{RMS}_{S}, \mathrm{RMS}_{\mathrm{L}}$, and LF, and higher values for the metrics $\alpha_{\mathrm{L}}$ and $\operatorname{HF}(11,28,29,31)$.

\section{MRI}

MRI was performed on a 3T scanner (Discovery MR750, GE Healthcare, Milwaukee, WI). In surviving infants, imaging was performed at the target age of 3-5 and 10-12 days as per our institutional protocol. An experienced neuroradiologist (G.V.) who was blinded to each infant's clinical status reviewed the MRI scans and scored the images according to a previously defined system (4). The degree of BG injury was assigned a score from 0 to 4 , and the degree of cortical WS injury was assigned a score from 0 to 5 . From these scores, infants were classified according to the predominant pattern of injury as previously described (5): total brain injury (maximum BG and WS score), BG-predominant (BG $\geq W S$ ) injury, or WS-predominant (WS $>B G$ ) injury. The WS group was further divided into infants with pure cortical/WMI and infants with mixed WS/mild BG injury. Infants who died prior to hospital discharge were grouped separately. Because of the small number of patients with global injury $(n=3)$, these patients were grouped with the $\mathrm{BN}$ predominant infants for the purposes of analysis as these patterns of injury have been linked to more severe outcome phenotypes. The score from the later MRI was used for classification of injury pattern, unless only the early MRI was completed.

\section{Statistical Analysis}

Clinical characteristics were analyzed using the standard measures of central tendency. HRV metrics were compared across the five brain injury pattern groups $(0=$ no injury, $1=$ pure cortical/WMI, $2=$ mixed $\mathrm{WS} /$ mild $\mathrm{BG}, 3=$ predominant $\mathrm{BG}$ or global injury, and $4=$ died) using Kruskal-Wallis tests. In addition, a random-effects mixed model was performed in order to adjust for the repeated measures in each subject and six of the most relevant covariates: gender, gestational age at birth, birth weight, encephalopathy grade, seizures during the study period, and use of vasopressors. The HRV metrics were log-transformed to satisfy the normality assumption. Secondarily, a sensitivity analysis was performed excluding patients with documented electrographic seizures during $24-27 \mathrm{~h}$ of life $(n=9)$, as there is evidence that there are heart rate characteristic changes around the time of seizures $(32,33)$. For those metrics that were significantly associated with brain injury pattern, we also performed receiver operating curve analyses to establish HRV cutoff points that could distinguish infants who died or had significant BG injury (groups 3-4) from those without BG injury (groups 0-1). We analyzed data from 74 infants prospectively enrolled in a study by evaluating biomarkers of brain injury in HIE. For these analyses, this sample size proved to have 0.87 power to detect significant associations $(P<0.001)$ between brain injury pattern and all detrended fluctuation analysis metrics and spectral analysis metrics. Statistical analyses were performed using SAS 9.3 (SAS Institute, Cary, NC).

\section{STATEMENT OF FINANCIAL SUPPORT}

This work was supported by the Clinical and Translational Science Institute at Children's National (UL1TR000075 and 1KL2RR031987-01) and the Intellectual and Developmental Disabilities Research Consortium (NIH P3OHD040677).

\section{DISCLAIMER}

The sponsors had no role in the design and conduct of the study, in the collection, management, analysis, and interpretation of the data, or in the preparation, review, or approval of the manuscript. 
Disclosure: The authors declare no conflict of interest.

\section{REFERENCES}

1. Shankaran S, Laptook AR, Ehrenkranz RA, et al. National Institute of Child H, Human Development Neonatal Research N. Whole body hypothermia for neonates with hypoxic-ischemic encephalopathy. N Engl J Med 2005;353:1574-84.

2. Ment LR, Bada HS, Barnes P, et al. Practice parameter: neuroimaging of the neonate: report of the quality standards subcommittee of the American Academy of Neurology and the Practice Committee of the Child Neurology Society. Neurology 2002;58:1726-38.

3. Executive summary: Neonatal encephalopathy and neurologic outcome, second edition. Report of the American College of Obstetricians and Gynecologists' Task Force on Neonatal Encephalopathy. Obstet Gynecol 2014;123:896-901.

4. Barkovich AJ, Hajnal BL, Vigneron D, et al. Prediction of neuromotor outcome in perinatal asphyxia: evaluation of MR scoring systems. AJNR Am J Neuroradiol 1998;19:143-9.

5. Miller SP, Ramaswamy V, Michelson D, et al. Patterns of brain injury in term neonatal encephalopathy. J Pediatr 2005;146:453-60.

6. Steinman KJ, Gorno-Tempini ML, Glidden DV, et al. Neonatal watershed brain injury on magnetic resonance imaging correlates with verbal IQ at 4 years. Pediatrics 2009;123:1025-30.

7. Shankaran S, Barnes PD, Hintz SR, et al. Brain injury following trial of hypothermia for neonatal hypoxic-ischaemic encephalopathy. Arch Dis Child 2012;97:F398-404.

8. Rutherford M, Ramenghi LA, Edwards AD, et al. Assessment of brain tissue injury after moderate hypothermia in neonates with hypoxicischaemic encephalopathy: a nested substudy of a randomised controlled trial. Lancet Neurol 2010;9:39-45.

9. Vergales BD, Zanelli SA, Matsumoto JA, et al. Depressed heart rate variability is associated with abnormal EEG, MRI, and death in neonates with hypoxic ischemic encephalopathy. Am J Perinatol 2014;31:855-62.

10. Goulding RM, Stevenson NJ, Murray DM, Livingstone V, Filan PM, Boylan GB. Heart rate variability in hypoxic ischemic encephalopathy: correlation with EEG grade and 2-y neurodevelopmental outcome. Pediatr Res 2015;77:681-7.

11. Massaro AN, Govindan RB, Al-Shargabi T, et al. Heart rate variability in encephalopathic newborns during and after therapeutic hypothermia. J Perinatol 2014;34:836-41.

12. Matic V, Cherian PJ, Widjaja D, et al. Heart rate variability in newborns with hypoxic brain injury. Adv Exp Med Biol 2013;789:43-8.

13. Schneebaum Sender N, Govindan RB, Sulemanji M, et al. Effects of regional brain injury on the newborn autonomic nervous system. Early Hum Dev 2014;90:893-6.

14. Shoemaker JK, Wong SW, Cechetto DF. Cortical circuitry associated with reflex cardiovascular control in humans: does the cortical autonomic network "speak" or "listen" during cardiovascular arousal. Anat Rec 2012;295:1375-84.

15. Cechetto DF. Cortical control of the autonomic nervous system. Exp Physiol 2014;99:326-1.

16. Okereafor A, Allsop J, Counsell SJ, et al. Patterns of brain injury in neonates exposed to perinatal sentinel events. Pediatrics 2008;121:906-14.
17. Rutherford M, Srinivasan L, Dyet L, et al. Magnetic resonance imaging in perinatal brain injury: clinical presentation, lesions and outcome. Pediatr Radiol 2006;36:582-92.

18. Harteman JC, Nikkels PG, Benders MJ, Kwee A, Groenendaal F, de Vries LS. Placental pathology in full-term infants with hypoxic-ischemic neonatal encephalopathy and association with magnetic resonance imaging pattern of brain injury. J Pediatr 2013;163:968-5.

19. Inder TE, Hunt RW, Morley CJ, et al. Randomized trial of systemic hypothermia selectively protects the cortex on MRI in term hypoxicischemic encephalopathy. J Pediatr 2004;145:835-7.

20. Iwata O, Thornton JS, Sellwood MW, et al. Depth of delayed cooling alters neuroprotection pattern after hypoxia-ischemia. Ann Neurol 2005;58:75-87.

21. Laptook AR, Shalak L, Corbett RJ. Differences in brain temperature and cerebral blood flow during selective head versus whole-body cooling. Pediatrics 2001;108:1103-0.

22. Perez A, Ritter S, Brotschi B, et al. Long-term neurodevelopmental outcome with hypoxic-ischemic encephalopathy. J Pediatr 2013;163: $454-9$.

23. Andriessen P, Oetomo SB, Peters C, Vermeulen B, Wijn PF, Blanco CE. Baroreceptor reflex sensitivity in human neonates: the effect of postmenstrual age. J Physiol 2005;568:333-41.

24. Yiallourou SR, Witcombe NB, Sands SA, Walker AM, Horne RS. The development of autonomic cardiovascular control is altered by preterm birth. Early Hum Dev 2013;89:145-52.

25. Shankaran S, Barnes PD, Hintz SR, et al. Eunice Kennedy Shriver National Institute of Child H, Human Development Neonatal Research N Brain injury following trial of hypothermia for neonatal hypoxicischaemic encephalopathy. Arch Dis Child Fetal Neonatal Ed 2012;97: F398-404.

26. Ulusar UD, Govindan RB, Wilson JD, Lowery CL, Preissl H, Eswaran H. Adaptive rule based fetal QRS complex detection using Hilbert transform. Conf Proc IEEE Eng Med Biol Soc 2009;2009:4666-9.

27. Govindan RB, Al-Shargabi T, Metzler M, Andescavage NN, Joshi R, du Plessis A. A spike correction approach for variability analysis of heart rate sick infants. Physica A 2016;444:35-42.

28. Govindan RB, Massaro AN, Al-Shargabi T, et al. Detrended fluctuation analysis of non-stationary cardiac beat-to-beat interval of sick infants. EPL 2014;108:40005.

29. Govindan RB, Massaro AN, Niforatos N, du Plessis A. Mitigating the effect of non-stationarity in spectral analysis-an application to neonate heart rate analysis. Comput Biol Med 2013;43:2001-6.

30. Heart rate variability: standards of measurement, physiological interpretation and clinical use. Task Force of the European Society of Cardiology and the North American Society of Pacing and Electrophysiology. Circulation 1996;93:1043-65.

31. Malliani A, Pagani M, Lombardi F, Cerutti S. Cardiovascular neural regulation explored in the frequency domain. Circulation 1991;84:482-92.

32. Malarvili MB, Mesbah M, Boashash B. Time-frequency analysis of heart rate variability for neonatal seizure detection. Australas Phys Eng Sci Med 2006;29:67-72.

33. Doyle OM, Temko A, Marnane W, Lightbody G, Boylan GB. Heart rate based automatic seizure detection in the newborn. Med Eng Phys 2010;32:829-39. 